\title{
Biochemistry of cellular healing
}

Volume 5 Issue 2 - 2017

\section{Opinion}

Western medicine is known for its focus on managing symptoms. Alternative or complimentary medicine is known for its focus on identifying underlying causes of the symptoms, often in terms of nutrient deficiency; toxicity; gut issues; etc. However, we need to be able to determine what is going on at a cellular level so that we can address the underlying cellular malfunction more effectively.

Both health and disease are the result of a huge number of interplaying processes in the body. Everything that happens in the body is the result of an incredible interplay of compounds, cellular structures, organs and systems. From creation and development to maintaining and metabolizing to disease, dysfunction and healing, the body is dependent on a huge number of interactive processes.

Most compounds we find in the body actually perform a number of different functions, i.e., CoQ10 is required to synthesize ATP in the electron transfer chain (ETC) of the mitochondria but it is also an anti-oxidant; Nitric oxide is required for vasodilatation and also in the immune system; etc.

One of the most complex compounds in the body is glutathione. There are 8 different known glutathione compounds in the body and they are involved in a huge number of diverse functions. Glutathione is initially well known for its redox mechanism. It not only works with all the different types of free radicals; but will re-stabilize other anti-oxidants once utilized; and it works in the cell, the cell membrane and outside the cells.

It is also well known for its detox capacities and as such is involved in several different detox mechanisms in the liver, i.e., Phase I\&II detox for general detoxification processes; Phase I\&II\&III detox processes to regulate estrogen levels; etc. It is also involved in cellular detox throughout the body.

Glutathione, however, is also involved in supporting the growth and development of immune cells; as well as regulating balances between certain types of cells, i.e., T1 \& T2; it is involved in the respiratory system, i.e., the red blood cells cannot uptake or eliminate 02 or $\mathrm{C} 02$ without sufficient glutathione; it regulates the production of Nitric Oxide which is necessary for both vasodilatation and the immune system; both directly and indirectly involved in hormone regulation; protects both the mitochondria and the telomeres; provokes growth of new telomeres; transports amino acids in the cells; and much more.

In fact, due to its wide spread functionality throughout every cell, organ and system in the body; there are numerous researchers who have claimed that there isn't a disease or dysfunction in the body that isn't correlated with low levels of glutathione - whether it is a cause or a result of the dysfunctional process.

The regulation and production of glutathione in any cell is dependent on three particular cellular processes: DNA expression; mitochondria production; and the methylation cycle. If there are challenges with any of these cellular processes, it will affect both the regulation and the production of glutathione.

\author{
Holly Fourchalk \\ Choices unlimited for Health \& Wellness Ltd, Canada
}

Correspondence: Holly Fourchalk, Choices unlimited for Health \& Wellness Ltd, Canada, Tel (604) 764-5203, Email holly@choicesunlimited.ca

Received: November 20, 2016 | Published: January 24, 2017
The methylation cycle, which consists of the methionine cycle; the folate cycle; the biopterin cycle and the urea cycle; are all required and work interactively together to synthesize the glutathione compounds. However, none of the cycles can function unless the mitochondria can provide sufficient ATP for fuel. Further, if the methylation cycle is working and the mitochondria are working but the DNA is not expressing the required RNA then the cells, again, cannot regulate and/or synthesize any of the glutathione compounds.

Of course, we can go back even further. In order for each of these cellular systems to function effectively, we need a healthy gut to metabolize and absorb the nutrients required to support each of these cellular systems. Everything from: a dysfunctional gut immune system; an imbalance in the gut microbiota; leaky gut syndrome; metal toxicity; a diet lacking nutrients; and/or a diet abundant in herbicides, pesticides, insecticides, POPs (Persistant Organic Pollutants), free radicals, artificial / synthetic / processed / micro-waved food toxins; etc can effect the metabolic efficiency of the gut and thus the necessary nutrients required for: gut/body absorption / transportation / metabolism / cellular absorption / metabolism \& functioning.

One important aspect of research is identifying how many different ways a given system can compensate for a lack of a given nutrient. For instance, utilizing choline when there is a deficiency of Vitamin B9 (folate). Yet, for a system to operate optimally, we want to provide it with all the nutrients it requires.

There are numerous companies who have produced products that effect the regulation or synthesis of glutathione. Unfortunately, these companies focus on specific components of the glutathione system rather than encompassing the entire underlying system required to regulate and synthesize glutathione. For instance, one of the first companies to provide supplements to support glutathione synthesis, focused on the ability to stabilize cysteine, one of the three amino 
acids in the glutathione compound, in the body, and thus we have $\mathrm{N}$-acetyl-cysteine. Some companies focus on providing the nutrients required synthesizing the end result glutathione compound, and thus we have MAX GXL. Some companies focus on turning on the necessary DNA required to synthesize glutathione, and thus we have Protandim. Some companies focus on the health of the mitochondria, and thus we have products like Mito Amp \& Mito Life.

However, as already identified all the interactive cellular processes are required and interdependent in the regulation and production of glutathione. If any one of them fails to function effectively, there will be issues with the regulation and synthesis of glutathione. Consequently, while each of the above identified products may contribute to enhancing glutathione production, none of the products are addressing the entire glutathione process. Unfortunately, it is like most diseases, there can be any number of issues causing the underlying issue.
Thus taking supplements/herbs/food that is shown to "turn on" given DNA may be great if the DNA is the weak link in the system. However, if it is the mitochondria that are causing the problem, then the client requires a different product.

\section{Acknowledgments}

None.

\section{Conflicts of interest}

Author declares there are no conflicts of interest.

\section{Funding}

None. 